\title{
Bicycle Disc Brake Thermal Performance: Combining Dynamometer Tests, Bicycle Experiments, and Modeling ${ }^{\dagger}$
}

\author{
Ioan Feier *, Joseph Way and Rob Redfield \\ Department of Mechanical Engineering, U.S. Air Force Academy, Air Force Academy, CO 80840, USA; \\ C21Joseph.Way@usafa.edu (J.W.); Rob.Redfield@usafa.edu (R.R.) \\ * Correspondence: Ioan.Feier@usafa.edu; Tel.: +1-719-333-1315 \\ + Presented at the 13th Conference of the International Sports Engineering Association, Online, \\ 22-26 June 2020.
}

Published: 15 June 2020

\begin{abstract}
High-power bicycle disc braking can create excessive temperatures and boiling brake fluid, resulting in performance degradation and damage. The goal of this work is to understand brake friction performance and thermal behavior for bicycle disc brakes. A previously described disc braking dynamometer is used to assess brake pad performance of sintered metallic brake pads, organic brake pads, and 'power' organic pads in up to $400 \mathrm{~W}$ of braking power. The friction coefficient is found to be dependent on both temperature and normal force. Friction curve fits are provided for temperatures between $300 \mathrm{~K}$ and $550 \mathrm{~K}$. Organic and 'power' organic pads are found to have similar behavior, and have higher friction coefficients compared to metallic pads. Further, brakes on an instrumented bicycle are tested in outdoor field trials during downhill descent. A MATLAB thermal model successfully predicts the downhill field brake disc temperatures when using the friction data curve fits.
\end{abstract}

Keywords: bicycle; braking; dynamometer; thermal; MATLAB; transient; model; numerical; disc; ride; friction; heat

\section{Introduction}

The goal of this work is to understand bicycle brake pad friction for several different types of bicycle brake pads (metallic, organic, and 'power' organic). The choice of friction material depends heavily on the course type, and the brake use needed for the given course. A process is presented where course data (via data logging velocity and elevation from a smartphone, for instance) is used to determine the bike's energy state, and corresponding instantaneous braking power. Alternatively, braking power is determined from recorded bicycle speed and brake line pressure, by using the friction coefficient data obtained by dynamometer tests. The braking power from either method is then entered into a MATLAB numerical heat transfer simulation to predict a braking temperature history. Combining friction data from dynamometer tests, energy and heat transfer models, and outdoor bicycle experiments enable a better understanding of braking systems.

\section{Materials and Methods}

\subsection{Experimental Dynamometer}

A previously documented brake dynamometer [1] has been upgraded to include a larger geared electric motor, a brake line pressure transducer, and the ability to convectively heat the brake disc. The updated experimental setup is shown in Figure 1. The geared electrical motor turns the bicycle 
disc brake through a torque sensor. The disc rotational speed is measured by a slotted optical sensor, and a hydraulic caliper and brake pad assembly provide the braking torque. The hydraulic pressure is recorded via a pressure transducer. A multichannel data acquisition system records sensor values at $25 \mathrm{~Hz}$.

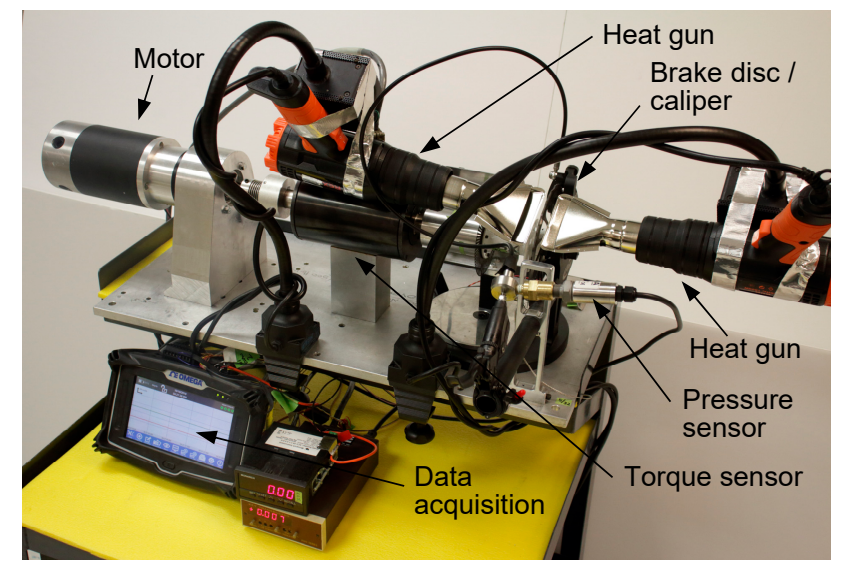

(a)

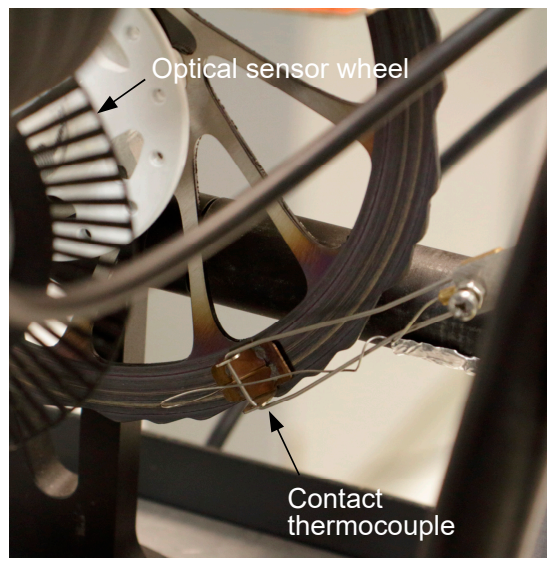

(b)

Figure 1. Brake dynamometer experimental setup: (a) Overall setup and data collection equipment; (b) the brake disc and contact thermocouple.

The dynamometer setup allows the torque, rotational speed, disc temperature, and brake line pressure to be recorded for various levels of braking. Tests were conducted by starting at room temperature and increasing brake pressure manually until the maximum achievable braking power was reached (approximately $350 \mathrm{~W}$ ), after which brake pressure was reduced to zero, and the process repeated. As the cycles accumulated, the brake disc temperature increased. Tests were halted at approximately $550 \mathrm{~K}$ in order to prevent boiling of the brake fluid. The test data were converted into calculated braking power, normal brake pad force, and braking force at the brake pads.

\subsection{Bicycle Testing}

A mountain bicycle was outfitted with a smartphone data logger (for GPS speed and barometric pressure), a front brake line pressure transducer, the sliding thermocouple from the dynamometer tests and a secondary data logger for the transducer and thermocouple. The power organic pads and corresponding rotor from the brake dynamometer test were used for outdoor testing, due to their good friction performance. Brake pressure and disc rotor temperature were recorded as the rider coasted downhill and applied the front brake. The $1.6 \mathrm{~km}$ course had $80.8 \mathrm{~m}$ of elevation decrease from start to finish (5\% grade). Trials were conducted during periods of low wind and without pedaling with a combined bicycle and rider mass of $90.7 \mathrm{~kg}$.

\subsection{Numerical Model}

A previously documented transient MATLAB thermal model [1] for bicycle braking was enhanced to include the ability to import braking power data from the outdoor bicycle brake tests (see Section 2.2). The MATLAB thermal model requires brake thermal parameters (convective coefficients, disc geometry) and transient brake power data. It outputs a prediction of bicycle disc temperature and the relative contribution of the disc, disc spokes, and brake pad heat rejection capability. Convective coefficients and methods for accounting for disc geometry and heat transfer modeling between components were already derived and presented in the prior work [1]. The disc convective coefficients from the prior work are used here with a modification to account for air density using a Reynolds number to the $4 / 5$ th power dependence. The braking power Pbrake during a time interval $\Delta t$ for outdoor tests was calculated using one of two methods: (Method 1) via calculation from brake pressure data, measured speed, and dynamometer friction data, and (Method 2) 
determined from a bicycle and rider system energy balance. The energy balance equation is similar to other energy models [2]:

$$
\begin{gathered}
\Delta E_{\text {potential }}+\Delta E_{\text {kinetic }}+\Delta E_{\text {inertial }}+\Delta P_{\text {rolling resistance }} \Delta t+\Delta P_{\text {air resistance }} \Delta t+\Delta P_{\text {avg brake }} \Delta t=0 \\
m g\left(h_{t}-h_{t+\Delta t}\right)+\frac{1}{2} m\left(V_{t+\Delta t}^{2}-V_{t}^{2}\right)+\frac{1}{2} I\left(\Omega_{t+\Delta t}^{2}-\Omega_{t}^{2}\right)-F_{\text {rolling }}\left(\frac{V_{t+\Delta t}+V_{t}}{2}\right) \Delta t \\
-\frac{1}{2} \rho\left(\frac{V_{t+\Delta t}+V_{t}}{2}\right)^{3} C_{D} A \Delta t-P_{\text {brake }} \Delta t=0
\end{gathered}
$$

Above, $m$ is mass $(\mathrm{kg}), g$ is the local acceleration due to gravity $\left(\mathrm{m} / \mathrm{s}^{2}\right), h$ is the bicycle elevation (m), $V$ is the velocity $(\mathrm{m} / \mathrm{s}), I$ is the total wheel rotational inertia $\left(\mathrm{kg} \cdot \mathrm{m}^{2}\right), \Omega$ is the angular acceleration $(\mathrm{rad} / \mathrm{s}), F_{\text {rolling }}$ is the rolling resistance force $(\mathrm{N}), \rho$ is air density $\left(\mathrm{kg} / \mathrm{m}^{3}\right), C_{D}$ is drag coefficient, and $A$ is bicycle/rider frontal area $\left(\mathrm{m}^{2}\right)$. The rolling resistance force is $F_{\text {rolling }}=C_{R} m g=\left(3.67 \cdot 10^{-4}\left(\left(V_{t+\Delta t}+\right.\right.\right.$ $\left.\left.\left.V_{t}\right) / 2\right)^{2}+7.213 \cdot 10^{-3}\right) \mathrm{mg}$, where the expression was obtained from existing literature for $200 \mathrm{kPa}$ knobby mountain bike tires on a road surface [3], and was normalized based on vertical load for use in this work. For a $5.5 \mathrm{~m} / \mathrm{s}$ speed with an $85.5 \mathrm{~kg}$ bike and rider combined mass (nominal value in the reference), for example, the expression predicts a rolling resistance force of $15.4 \mathrm{~N}$. This would be a coefficient of rolling resistance $C_{R}$ for this speed of 0.0183 , which agrees well with other published results for knobby tires on asphalt (i.e., $C_{R}=0.013-0.017$ from di Prampero [4]). The $\left(V_{t+\Delta t}+V_{t}\right) / 2$ terms that are in the above expressions are used to average the velocity between two time steps $t$ and $\Delta t$ to better estimate forces. Based on laboratory-measured wheel inertia tests, a value of $0.145 \mathrm{~kg} \cdot \mathrm{m}^{2}$ per wheel was used for wheel rotational inertia. For drag, a $C_{D} A$ value of $0.357 \mathrm{~m}^{2}$ was chosen from the literature [3].

\section{Results}

Results from the dynamometer friction tests, the outdoor bicycle braking tests and the numerical model are presented below. Dynamometer friction data is presented for metallic pads, organic pads, and the power organic pads. An example outdoor test, and numerical model thermal prediction is provided for the power organic pads. Prior work [1] only validated the thermal model using the dynamometer, but presented no friction data or model comparison to field tests.

\subsection{Friction Data}

Figure 2a shows typical time histories of dynamometer sensor data, including calculated braking power. Note the gradual disc temperature rise throughout the test. Figure $2 \mathrm{~b}$ shows the data converted into a friction force surface that is a function of normal pad force and pad temperature. Both Figure 2a,b correspond to the same power organic pad test. As it is difficult to place a load cell or force transducer between the caliper pistons and brake pads, the normal pad force was calculated based on line pressure and caliper piston area. Caliper piston seal stiction or piston seal elastic force is thus included in the normal force, and this may explain some of the friction force nonlinearity at low normal forces. Therefore, the friction force surfaces presented here are for the brake pads and caliper as a system.

A fitting function was desired (useful for engineering analysis, thermal modeling, and comparison) to model the friction behavior as a function of normal pad force and temperature for each pad. The fitting function that was chosen for the friction force, $F_{\text {friction }}(\mathrm{N})$, response surface is:

$$
F_{\text {friction }}=\operatorname{MAX}\left\{\left[a_{1}+a_{2} F_{N}+a_{3} \cos \left(w_{1} T\right)+a_{4} \sin \left(w_{1} T\right)\right],\left[a_{5} F_{N}+a_{6} F_{N} *\left(T-a_{7}\right)^{2}\right]\right\}
$$

where $F_{N}$ is the brake pad normal friction force (in N), T is the temperature (in K), and $a$ and $w$ are fitting coefficients. The arguments of the cosine and sine functions are in radians. The fitting function is physically realistic as it is guaranteed to have a friction force of zero when the normal force is zero. The $M A X\{[A],[B]\}$ function chooses the maximum of the two arguments in the brackets: $[A]$ and $[B]$. The first equation $[A]$ models the high braking force slope, whereas the second equation $[B]$ corresponds to low values of braking force, below approximately $10 \mathrm{~N}$ of friction. This piecewise 
definition is a necessary compromise to blend in $F_{\text {friction }}$ behavior, because the friction coefficient $\left(\mu=F_{\text {friction }} / F_{N}\right)$ depends strongly on the normal force $F_{N}$ at low braking pressures. The equation above was used to fit the friction data for each pad that was tested. The gray surface in Figure $2 \mathrm{~b}$ corresponds to the power organic curve fit, for instance. Table 1 below shows the resulting curve fit parameters and the quality of fit for the three different pad types.

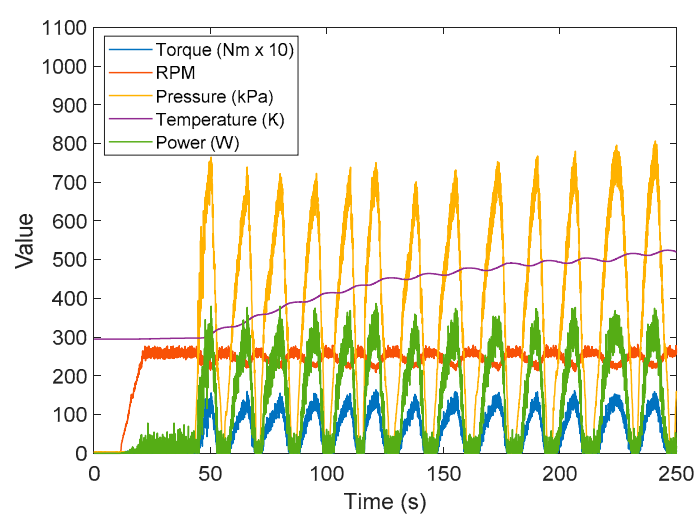

(a)

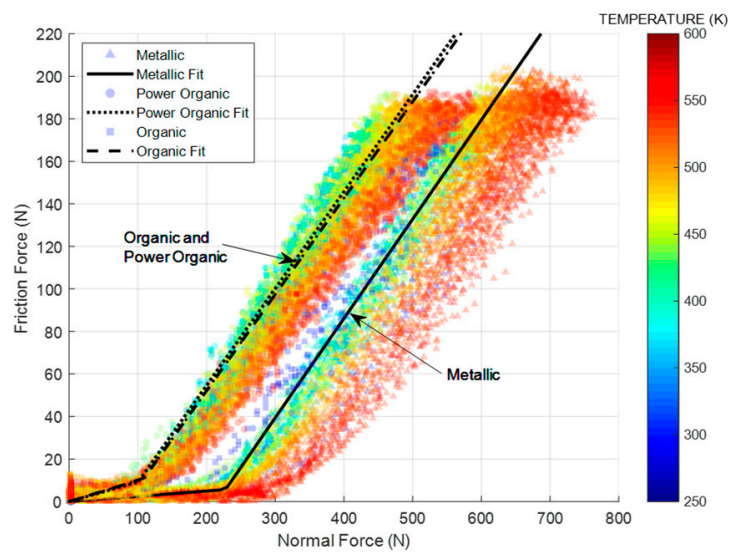

(c)

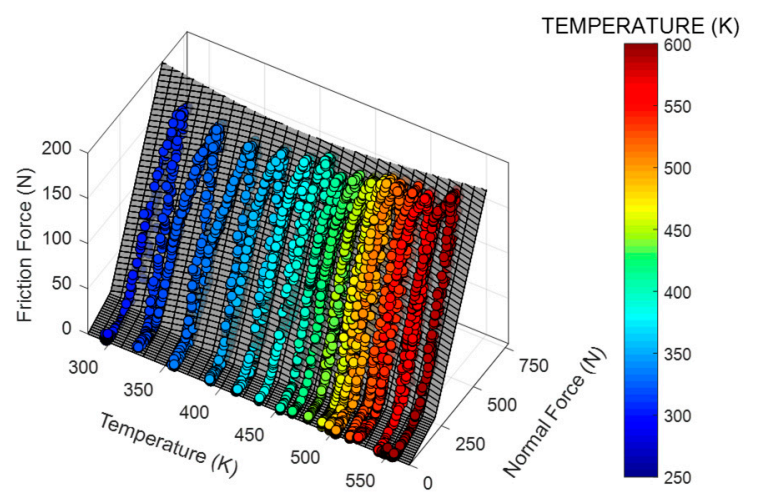

(b)

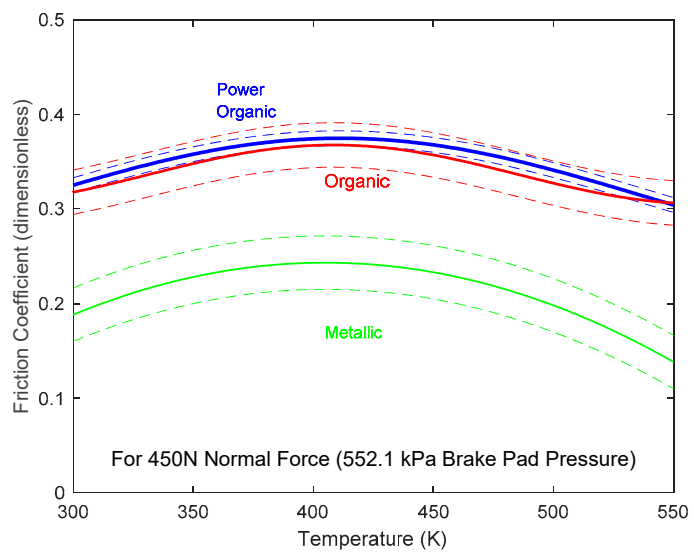

(d)

Figure 2. (a) Example dynamometer transient experimental data for power organic pad friction test. Power is calculated, and all other parameters are measured; (b) Example experimental data points (colored by temperature) and curve fitted friction response surface (gray) for the power organic pad from room temperature to approximately $560 \mathrm{~K}$; (c) Scatter plot of the metallic, organic, and power organic pad experimental friction forces, colored by temperature, with curve fits superimposed at 408 K; (d) Friction coefficients vs. temperature as extracted from the curve fitted response surface for each pad (for $450 \mathrm{~N}$ normal force on a total pad area of $815 \mathrm{~mm}^{2}$, or $552.1 \mathrm{kPa}$ equivalent pad normal pressure), with error limits shown as dashed lines based on the curve fit RMSE values.

Table 1. Curve fitting coefficients for Equation (3) for the three types of brake pads that were tested. The quality of fit for the upper equation [A], which accounts for most of the data, is shown via $\mathrm{R}^{2}$ (i.e., R-Square; coefficient of determination) and RMSE (Root mean squared error or also known as fit standard error).

\begin{tabular}{ccccccccccc}
\hline Pad Type & $\boldsymbol{a}_{\mathbf{1}}$ & $\boldsymbol{a}_{\mathbf{2}}$ & $\boldsymbol{a}_{\mathbf{3}}$ & $\boldsymbol{a}_{\mathbf{4}}$ & $\boldsymbol{a}_{\mathbf{5}}$ & $\boldsymbol{w}_{\mathbf{1}}$ & $\boldsymbol{a}_{\mathbf{6}}$ & $\boldsymbol{a}_{\mathbf{7}}$ & $\mathbf{R}^{\mathbf{2}}$ & RMSE \\
\hline Metallic & -4069 & 0.4668 & 3605 & 1659 & 0.02475 & 0.001065 & 0.000 & 406 & 0.956 & 12.7 \\
Organic & -52.2 & 0.4532 & -6.786 & 12.23 & 0.09995 & 0.02047 & 0.000 & 408 & 0.951 & 10.6 \\
Power Organic & -63.85 & 0.4561 & 11.38 & -24.74 & 0.09115 & 0.01251 & $-2.261\left(\times 10^{-7}\right)$ & 410 & 0.995 & 3.52 \\
\hline
\end{tabular}

Figure 2c shows a comparison between the three different pad types as normal force $F_{N}$ is increased. The data is colored by temperature. Figure $2 b$ is a side view of Figure $2 b$, but for all pad types. Solid, dotted, and dashed black lines show the curve fit equations evaluated at $408 \mathrm{~K}$ (i.e., near 
peak friction temperature). The metallic pads require greater $F_{N}$ to achieve $F_{\text {friction }}$ values comparable to the organic or power organic pads. Figure $2 \mathrm{~d}$ shows an example of temperature behavior that can be derived from the curve fit surfaces. All pads showed a peak friction coefficient between $406 \mathrm{~K}$ and $410 \mathrm{~K}$. These intermediate temperatures have the highest friction coefficient $(\mu=$ $F_{\text {friction }} / F_{N}$ ) for all pads. Within the accuracy of the dynamometer experiments, the organic and power organic pads had equivalent behavior, and both showed higher friction performance compared to the metallic pads. Limited data exist for bicycle disc brake pad friction coefficients, although some does exist for rim brakes suggesting a friction coefficient of 0.4 [5].

\subsection{Bicycle Testing and Numerical Modeling Thermal Results}

Figure 3a compares the ability of the numerical model to match experimental temperatures from the sliding thermocouple during a downhill descent. Predictions from the numerical model are shown for braking power calculated using the two methods already mentioned: (1) brake line pressure, bicycle speed and curve fit friction data; and (2) bicycle speed and elevation (energy model from Equation (1)). Figure $3 \mathrm{~b}$ shows a comparison of the input braking power and the power dissipation from various front brake surfaces.

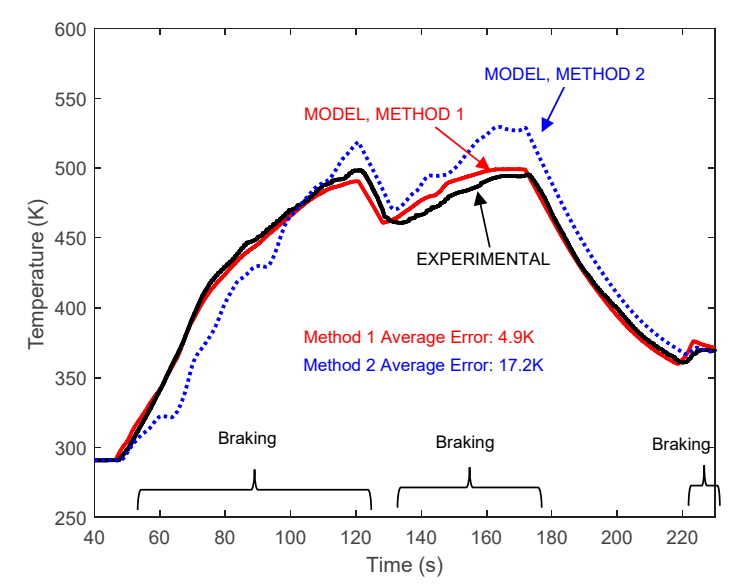

(a)

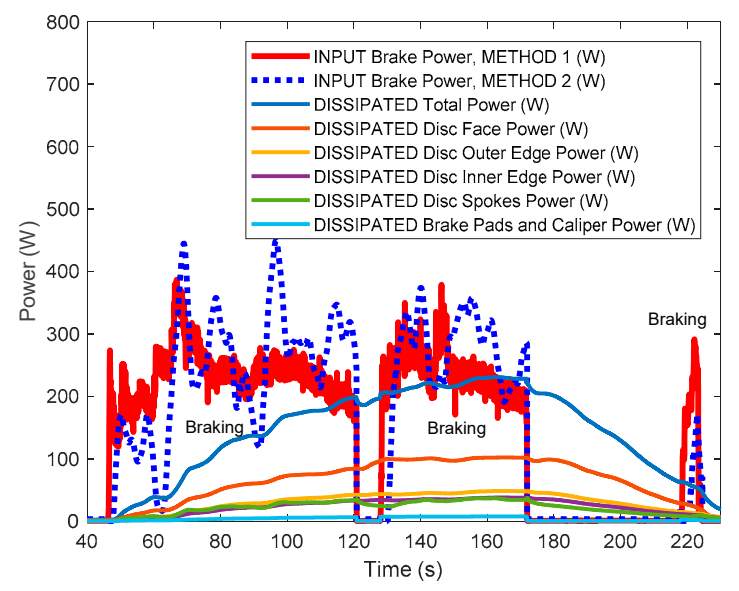

(b)

Figure 3. (a) Transient numerical modeling results from a downhill test showing temperature history for several braking events, with models shown for braking power determined via Method 1 (pressure, speed, and friction data) and via Method 2 (speed and elevation data), and compared to experimental data with total average errors indicated $\int\left(\left|T_{\text {model }}-T_{\text {experiment }}\right|\right) d t / t_{\text {total }} ;(\mathbf{b})$ Input brake power for Methods 1 and 2, and example capability of the model to predict dissipated power for different disc surfaces (dissipated power is shown only for Method 1).

\section{Discussion}

Method 1 for calculating the brake input power for the MATLAB thermal model has an excellent match to the experimental disc temperature (within $4.9 \mathrm{~K}$ on average). The temperature match independently validates the friction data curve fits obtained on the dynamometer in this work. Additionally, the agreement of the model temperature to the experimental temperature validates the thermal modeling process described in prior work [1], but now for field conditions. The thermal model predicts that the majority of heat dissipation is from the brake disk surfaces, followed next by the disc spokes (connecting the disc to the hub), and then followed by the brake pads and caliper.

Method 2 (speed and elevation coupled to the energy model) qualitatively matches experimental behavior. Better matching could be achieved for Method 2 by measuring aerodynamic and rolling resistance for the specific bicycle/rider. The GPS speed inaccuracy and low refresh rate $(1 \mathrm{~Hz}$ update limitation on most smartphones) contribute to the uncertainty in the braking power estimation from the dynamic bicycle energy model, and consequently, the predicted temperature. The error in power estimation is magnified because the kinetic energy term depends on $V^{2}$ and the aerodynamic/rolling 
resistance terms depend on $V^{3}$. To reduce error, an alternative speed measurement can be used for Method 2, such as a wheel speed encoder that has a more accurate measurement of speed. Note that the GPS smartphone speed error was less significant for Method 1 as speed was only used to calculate brake power from the friction torque, a calculation which is linear in $V$.

\section{Conclusions}

Combining dynamometer tests, numerical thermal models, and bicycle tests demonstrate a methodology to systematically predict brake temperatures and friction performance. A combined workflow is suggested where data from a bicycle ridden on the course of interest can be recorded via a smartphone or data acquisition system, and then combined with dynamometer-measured friction coefficients and a numerical heat transfer code in order to predict brake temperatures. For example, the MATLAB thermal model combined with field elevation history, field brake line pressure history, and brake pad/caliper friction data can successfully predict transient disc temperature for multiple sequential braking events.

Acknowledgments: This research project has been performed at the U.S. Air Force Academy. No external funding for research or publication has been received. The authors would like to acknowledge SRAM LLC for bicycle braking discussions and for loaning various brake components for the testing.

Conflicts of Interest: The authors declare no conflict of interest. The funding sponsors had no role in the design of the study; in the collection, analyses, or interpretation of data; in the writing of the manuscript, and in the decision to publish the results.

\section{References}

1. Feier, I.; Redfield, R. Thermal/Mechanical Measurement and Modeling of Bicycle Disc Brakes. Proceedings 2018, 2, 215, doi:10.3390/proceedings2060215.

2. Martin, J.C.; Milliken, D.L.; Cobb, J.E.; McFadden, K.L.; Coggan, A.R. Validation of a Mathematical Model for Road Cycling Power. J. Appl. Biomech. 1998, 14, 276-291, doi:10.1123/jab.14.3.276.

3. Bertucci, W.M.; Rogier, S.; Reiser, R.F. Evaluation of aerodynamic and rolling resistances in mountain-bike field conditions. J. Sports Sci. 2013, 31, 1606-1613, doi:10.1080/02640414.2013.792945.

4. Di Prampero, P.E. Cycling on Earth, in space, on the Moon. Eur. J. Appl. Physiol. 2000, 82, 345-360, doi:10.1007/s004210000220.

5. Keong, L., Teoh, C. Topology Analysis of Bicycle Rim Brake Pad to Improve Braking Performance. MATEC Web Conf. 2018, 217, doi:10.1051/matecconf/201821704004.

This material is declared a work of the U.S. Government and is not subject to copyright protection in the United States; DISTRIBUTION A. Approved for public release: Distribution unlimited. Foreign copyrights may apply. @ 2020 by the authors. Licensee MDPI, Basel, Switzerland. This article is an open access article distributed under the terms and conditions of the Creative Commons Attribution (CC BY) license (http://creativecommons.org/licenses/by/4.0/). 